Methods This study was conducted in a specialist palliative care unit with 20 inpatient beds and day therapy unit. Discussions were held with the counselling, medical and nursing staff to develop the support service. A survey was distributed to MDT members, giving 24 anonymous responses. Subsequently, a monthly emotional support forum was piloted, facilitated by a ward nurse and supported by the counsellors and ward manager. A follow-up survey was distributed 16 months later with 32 respondents.

Results The initial survey identified staff may benefit from emotional support, with over 50\% experiencing disturbed sleep, intrusive thoughts and feeling sad or depressed at some point over the past month. Preferred options were mindfulness sessions and an emotional support forum. Over $70 \%$ of respondents to the second survey attended the forum at some point. Of those, $85 \%$ felt it an inclusive and safe environment and $74 \%$ found it helpful. $48 \%$ could not attend as often as hoped, predominantly due to time constraints and staffing. There were no significant differences between measures of anxiety and depression before and after forum introduction.

Conclusions Staff were more aware of the counselling service to support patients/relatives than themselves, but many engaged with the forum and found it helpful. Areas for improvement include increasing awareness and facilitating forum attendance. This study was not designed to detect objective changes in anxiety/depression, however, our qualitative findings support the forum as a low cost and convenient approach to improving staff wellbeing.

\section{HEART FAILURE AND PALLIATIVE CARE: AN INTEGRATED SERVICE FOR PATIENTS ACROSS HOSPITAL AND COMMUNITY SETTINGS}

JR Ross, F Hodson, S Karwatowski, M Albarjas, R Nash, J Smith, R George. St Christopher's Hospice, King's College Hospital, Princess Royal University Hospital

\subsection{6/bmjspcare-2019-ASP.17}

Background An integrated clinical service for patients with end-stage heart failure (HF) was funded by Bromley Clinical Commissioning Group; Bromley has no community HF nurse specialists.

Method Six GP practices participated and referral criteria were agreed. Patients were assessed and followed up by an Advanced Nurse Practitioner, with experience in HF and palliative care (PC), who worked closely with cardiology consultants and HF/PC hospital teams, the Community PC Team (St Christopher's Hospice) and primary care colleagues. Following initial assessment, patients were discussed at joint MDMs to assess both cardiac and palliative needs. We report data from the service evaluation of this clinical pilot.

Results 102 patients, mean age 84.2 \pm 10.2 years, were referred over 1 year. 89 were accepted. 55 (61.8\%) had LVSD, 14 (15.7\%) patients had HF with preserved ejection fraction, 13 (14.6\%) had predominately valvular dysfunction, 5 (5.6\%) had right sided HF. Patients had multiple co-morbidities, significant renal impairment and mental health diagnoses (depression and dementia). Two thirds required considerable assistance or were immobile $>50 \%$ of the time, with $18 \%$ predominately bedbound. 29 (33\%) lived alone, 49 (55\%) with family, and 12\% in care homes. The majority of carers were female (86\%), $25 \%$ of carers frequently/nearly always 'did not have time for themselves' with $40 \%$ reporting a significant impact of caring on their own health. Meta-Analysis Global Group in Chronic Heart Failure (MAGGIC) scores were not accurate in predicting outcome. $44 \%$ of patients died during the pilot. Of these, $69 \%$ died at home/hospice $(41 \% / 28 \%)$ versus $31 \%$ in hospital. Further PROMs including integrated palliative care outcome scale (iPOS) will be presented.

Conclusion This pilot was positive and demonstrates benefits and challenges of joint working between integrated teams. We will discuss how ongoing integrated practice can be developed locally with dissemination of good practice and joint working to other groups.

\section{Free Papers $19-21$ | Training}

\section{DO PALLIATIVE CARE CHAMPIONS INCREASE STAFF CONFIDENCE TO HAVE DIFFICULT CONVERSATIONS?}

Jane Manson, Helen Blomfield, Louise Patterson, Angela Todd, Lisa Farndon. Sheffield Teaching Hospitals

\subsection{6/bmjspcare-2019-ASP.18}

Background Previous research and recent media coverage have identified that healthcare professionals who don't work in a palliative care setting do not feel comfortable talking to patients about palliative-related issues. As caseloads become more frail and complex, there is an increasing need for staff members at all levels to feel more confident discussing issues such as advanced life planning, deteriorating function, and future care needs. Champions have proven to be highly effective in knowledge translation in order to instigate positive changes in healthcare, however there is no evidence evaluating the 'champion approach' in palliative care or in a rehabilitation setting.

Aim To evaluate the introduction of the palliative care champion role on staff confidence and patient outcomes.

Methods A cohort study of four bed-based intermediate care units ( 2 intervention, 2 control) was used. Three champions based in the two intervention units received specialist training and disseminated this to the remaining cohort. This was followed by a 5 month data collection period.

Results Confidence of the intervention unit staff increased an average of 1.9 points on a scale of $1-10$ post training $(0.1$ control). This confidence continued to increase to an average of 2.4 points following the 5 month intervention period $(0.3$ control). There was no change to perceived contact with palliative patients or barriers to conversation.

Conclusion Introducing palliative care champions could be an easy, cost-effective way of increasing staff confidence to have difficult conversations with patients.

\section{TO EXPLORE THE UTILITY OF THE BREATHING, THINKING, FUNCTIONING (BTF) CLINICAL MODEL IN MANAGING BREATHLESSNESS THROUGH A PRACTICAL WORKSHOP AIMING TO EQUIP PROFESSIONALS}

Declan Cawley, Aileen McCartney, Anna Spathis. Wisdom Hospice, University of Cambridge

\subsection{6/bmjspcare-2019-ASP.19}

Background Breathlessness is a common and distressing symptom within progressive respiratory conditions. Emerging research advocates that irrespective of diagnosis, management 